\title{
High-density automated mapping and entrainment pacing for successful atypical flutter ablation with one RF application
}

\author{
Urszula Angelika Hangiel 1,B,D,F, Jacek Kuśnierz ${ }^{2, A-B}$, Paweł Derejko 2,A,C,E-F, Aleksander Bardyszewski²,B-C \\ Dobromiła Dzwonkowska ${ }^{2, \mathrm{~B}-\mathrm{C}}$ \\ A - Research concept and design, B - Collection and/or assembly of data, C - Data analysis and interpretation, \\ D - Writing the article, E - Critical revision of the article, F - Final approval of article \\ 1. Biosense Webster, J\&J Poland \\ 2. Cardiology Department, Medicover Hospital, Warsaw
}

Address for correspondence:

Urszula Angelika Hangiel, Biosense Webster, J\&J Poland

email: urszula.hangiel@gmail.com

Jacek Kuśnierz, Cardiology Department, Medicover Hospital, Warsaw

email: jakusnierz@interia.pl

Paweł Derejko, Cardiology Department, Medicover Hospital, Warsaw

email: pderejko@yahoo.com

Aleksander Bardyszewski, Cardiology Department, Medicover Hospital, Warsaw

email: albard2000@gmail.com

Dobromiła Dzwonkowska, Cardiology Department, Medicover Hospital, Warsaw

email: dobromila.dzwonkowska@medicover.pl

Received: 31.12.2017

Revised:

Accepted: 31.12.2017

Final review: 05.12.2017

DOI: $10.24255 / \mathrm{hbj} / 81640$

\section{Key words:}

atypical atrial flutter, catheter ablation, entrainment, high-density mapping, atrial fibrillation

\section{Abstract}

Atypical left atrial flutter may occur as a complication after atrial fibrillation ablation, especially when linear and substrate ablation were initially deployed. In such cases, the most effective therapy is radiofrequency ablation, but the procedure

\section{Case report}

A 43-year-old woman, after previous ablations for atrial fibrillation and typical atrial flutter, was scheduled for another ablation, due to symptomatic atypical atrial flutter. Echocardiography showed left atrial (LA) diameter within the normal range $(40 \mathrm{~mm})$ and normal ejection fraction $(65 \%)$.

Previously the patient underwent circumferential pulmonary vein isolation and linear ablation with lines in the cavo-tricuspid isthmus (CTI), left atrial roof and mitral isthmus groove. During the last ablation, bi-directional block was confirmed in all locations and no arrhythmia was induced with aggressive stimulation during isoproterenol infusion. can be long lasting and challenging. Use of multielectrode catheters and high-density mapping algorithms together with a conventional electrophysiological approach may shorten and simplify treatment.

The ECG recorded on admission suggested left atrial flutter with 2:1 conduction, with positive $\mathrm{F}$ waves in leads II, III, aVF, and V1 and negative in leads aVR and AVL (Fig. 1). Intracardiac signals (IC) showed atrial flutter with CL 295-300 ms, with proximal and distal bipoles at the catheter placed in the coronary sinus (CS) activated simultaneously.

Due to the electrographic pattern of arrhythmia and previous catheter ablations the operator (JK) decided to perform high-density mapping of left atrial endocardial activation using a multielectrode mapping (MEM) catheter (PentaRay, 20 poles, spacing 2-6-2, $1 \mathrm{~mm}$ width electrodes) and dedicated automatic algorithm ${ }^{[1]}$ (Confidense, Carto 3 Biosense Webster). 


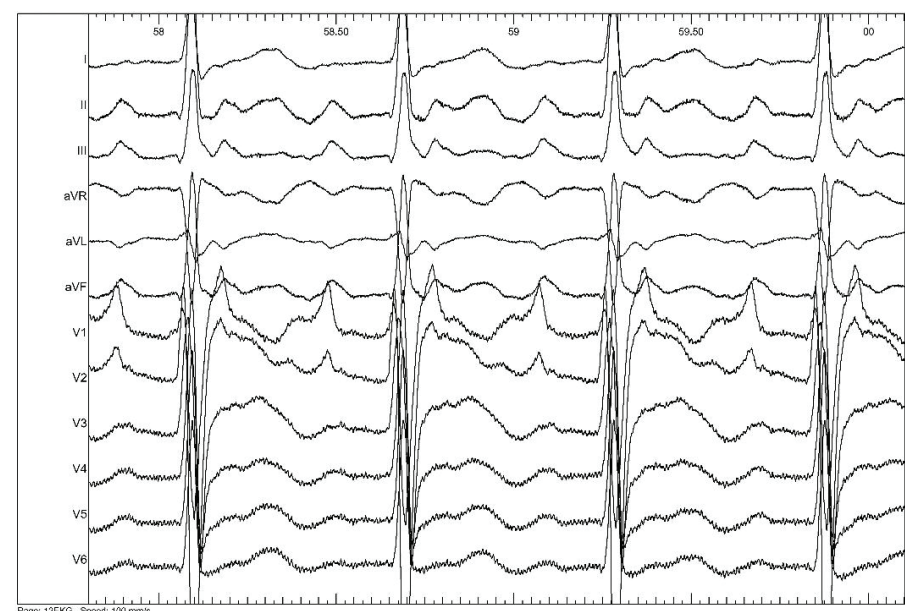

Figure 1. Baseline 12-lead ECG. Atypical flutter with positive $F$ waves in leads II, III, aVF, and $\mathrm{V} 1$ and negative in leads $\mathrm{aVR}$ and $\mathrm{aVL}$

After transseptal puncture, a 20 pole mapping catheter (PentaRay) and contact force sensing irrigated ablation catheter (SmartTouch, Biosense Webster) were inserted into the LA. Next, 3D reconstruction of the chamber and high-density mapping of flutter activation were performed, using the automated mode with the following settings: reference CS 3-4, minimum value, window of interest (WOI): -150; 145, CL stability: $5 \%$, LAT stability $3 \mathrm{~ms}$, catheter position stability $6 \mathrm{~mm}$ with respiratory gating, point density $1 \mathrm{~mm}$. To ensure that all points were collected in close proximity to the LA tissue, an additional filter - Tissue Proximity - was used.

The activation map covered almost the entire cycle length of the flutter. To display the activation pattern of the flutter the "early meets late" feature was used with default settings of the mapping system. The sensitivity level was set for $90 \%$ of CL.

Activation mapping indicated the perimitral circuit (Fig. 2). Post-pacing interval (PPI) measured from the CTI and the mitral isthmus anterior to the left inferior pulmonary vein were longer than the tachycardia cycle length (TCL) (PPI > TCL + 30 $\mathrm{ms})$. With the settings used, the "earliest" activation site was found below lower the border of the left inferior pulmonary vein (LIPV). This point corresponded with a low voltage $(<$ $0.2 \mathrm{mV}$ ) area at the inferior part of the LA posterior wall. PPI measured at that site after entrainment pacing (with CL 270 ms) was $306 \mathrm{~ms}$, almost equal to TCL (300 ms).

RF application at that point after 4 seconds prolonged CL of arrhythmia to $320 \mathrm{~ms}$ (Fig. 3a), and finally terminated it after 17 seconds (Fig. 3b).

After 20 minutes waiting time conduction block across all the lines performed, including the mitral isthmus line, was confirmed. Arrhythmia was non-inducible during control EPS using aggressive stimulation (burst pacing from coronary sinus catheter up to $220 \mathrm{~ms}$ ).

At 1-month follow-up the patient remains in sinus rhythm without antiarrhythmic drugs.

\section{Discussion}

Left atrial flutter tachycardia is a relatively frequent complication after atrial fibrillation ablation, especially when linear and substrate ablation were initially deployed ${ }^{[2]}$. Ablation of this arrhythmia can be challenging and in many patients several circuits coexist. When undertaking such a procedure, several issues should be considered. For localization of atypical reentrant circuits, the flutter wave morphology has limited utility. In the LA, in particular there is a large overlap in the flutter wave morphologies among different circuits ${ }^{[3]}$. In our patient the presumed exit site was below the LIPV, but flutter waves were positive in inferior leads (Fig. 1, 2). The most frequent
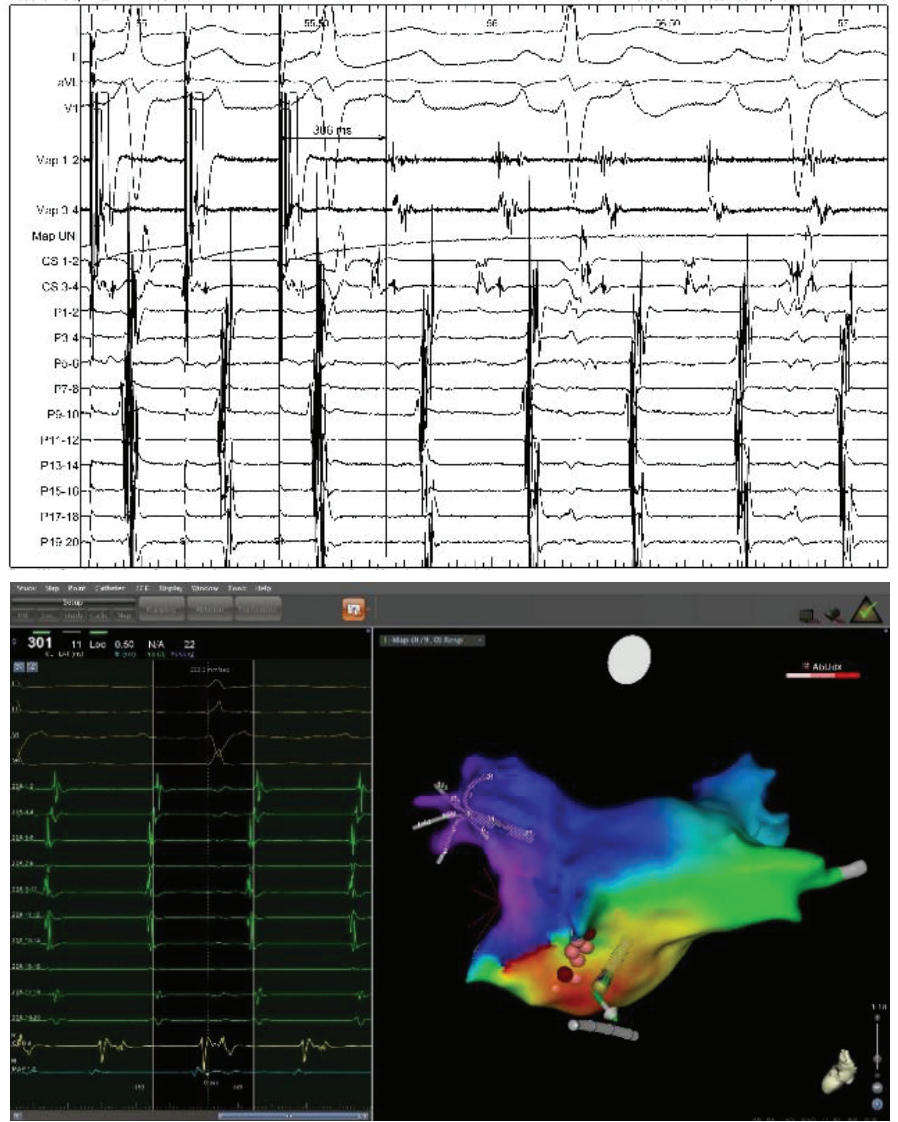

Figure 2. Entrainment pacing with cycle length $270 \mathrm{~ms}$ from inferior part of the left atrial posterior wall - pointed by tip of catheter. PPI - $306 \mathrm{~ms}$, AFL CL $300 \mathrm{~ms}$. Of note, during pacing activation at CS 3-4 is slightly different than during flutter, which indicates that CS 3-4 is captured antidromically. Therefore, it is not a case of true "concealed entrainment". It is due to proximity of the pacing site to CS 3-4 and probably the fact that pacing is performed from the "exit site" from the circuit. This is suggested by the fact that the local activation time at Map 1-2 (pacing site) precedes only slightly the beginning of the F-wave.

mechanism of such arrhythmia is macro-reentry, although focal or micro-reentrant arrhythmia can also occur. In focal and micro-reentry tachycardias $\mathrm{P} / \mathrm{F}$-wave durations are shorter and isoelectric intervals are longer than with macroreentry. In our patient the P/F-wave was relatively short (around $150 \mathrm{~ms}$ ), but the mechanism turned out to be macroreentrant ${ }^{[3]}$. Adenosine can be useful in distinguishing macroreentrant from focal atrial tachycardias. With rare exceptions adenosine does not terminate macroreentrant arrhythmias, while it terminates or suppresses focal tachycardia due to automaticity or triggered activity ${ }^{[4]}$. Evidence for a macroreentrant mechanism can be obtained through manifest entrainment and entrainment with concealed fusion; however, electroanatomic mapping can be very helpful and suggestive $\mathrm{e}^{[5]}$. Mapping of around $90 \%$ of atrial flutter CL strongly suggests a macroreentrant mechanism. However, it can be misleading, for example when the "focus" originates close to the existing line of block. 


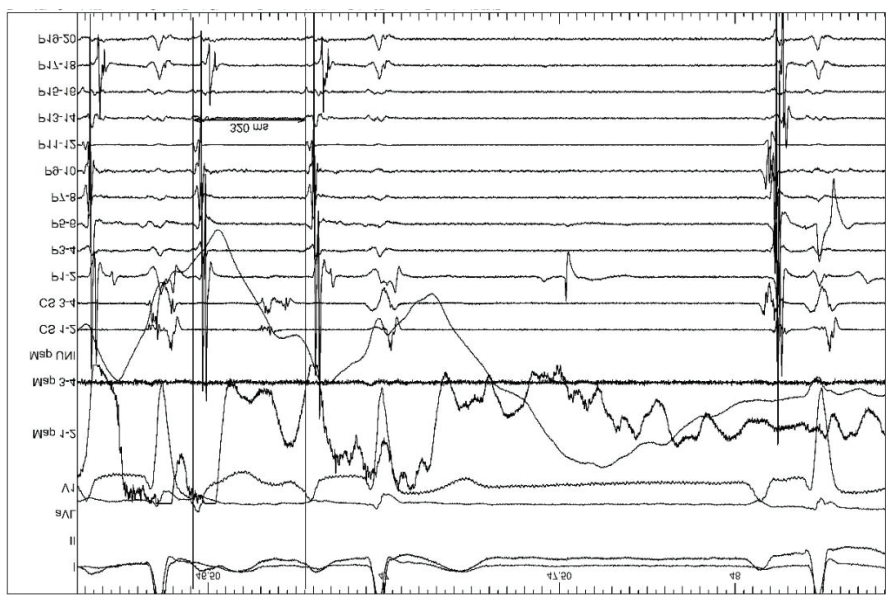

Figure 3. RF application, AFL CL prolongation from $300 \mathrm{~ms}$ to $320 \mathrm{~ms}$, sinus rhythm recovery.

Ablation of macroreentrant flutter requires finding the critical isthmus, which can be a narrow channel or a broad region ${ }^{[5,6]}$. When an electroanatomical map displays the activation sequence of macroreentrant tachycardia, we anticipate seeing an area where our earliest defined electrograms meet their latest counterparts ${ }^{[7]}$. However, in a reentry circuit there is neither an early nor a late point of activation, and thus the location of a site designated as early is simply a reflection of the arbitrarily chosen reference point. Most of all, the site where "early meets late" does not necessarily indicate the critical isthmus of the circuit ${ }^{[6]}$, as electroanatomical maps can be ambiguous or misleading. Thus, the presumed isthmus should be confirmed by entrainment mapping. However it must be kept in mind that the response to entrainment pacing can also be misleading, especially when the pacing cycle is short ${ }^{[8]}$. As information provided by electroanatomic mapping and entrainment pacing is complementary, both methods should be used if possible ${ }^{[1,9]}$.

The end-point of ablation in the case of atypical flutter should be bi-directional block across the ablation line or isthmus. Non-inducibility of arrhythmia is another endpoint; however, aggressive stimulation may induce non-clinical arrhythmias. For macroreentrant arrhythmias that occur after atrial fibrillation ablation, long-term success rates of 77 to $95 \%$ have been reported ${ }^{[10,11]}$, and some of the patients require multiple procedures to achieve stable sinus rhythm. This was the case in our patient.

\section{Conclusion}

Some patients scheduled for AF ablation may require multiple or staged procedures to achieve success. Extensive ablation for atrial fibrillation can create a substrate for other arrhythmias. In the case of complex arrhythmias a combination of high-density automated mapping and entrainment pacing allows for fast and efficient circuit identification and ablation.

\section{References}

1. El Hadad M., Houben R., Stroobandt R et al. Novel algorithmic methods in mapping of atrial and ventricular tachycardia. Circulation: Arrhythmia and Electrophysiology. 2014; 7:463-472,

2. Verma A, Mantovan R, Macle L, et al. Substrate and Trigger Ablation for Reduction of Atrial Fibrillation (STAR AF): a randomized, multicentre, international trial. European Heart Journal. 2010;31(11):1344-1356.

3. Buttà, C., Tuttolomondo, A., Giarrusso L, et al. Electrocardiographic diagnosis of atrial tachycardia: classification, P-wave morphology, and differential diagnosis with other supraventricular tachycardias. Ann Noninvasive Electrocardiol, 2015; 20(4): 314-327.

4. Markowitz SM, Stein KM, Mittal S, et al. Differential effects of adenosine on focal and macroreentrant atrial tachycardia. J Cardiovasc Electrophysiol. 1999 Apr; 10(4):489-502.

5. De Ponti R, Marazzi R, Zoli L, et al. Electroanatomic mapping and ablation of macroreentrant atrial tachycardia: comparison between successfully and unsuccessfully treated cases, J Cardiovasc Electrophysiol. 2010; 21(2):155-62

6. Ouyang F, Ernst S, Vogtmann T, et al. Characterization of reentrant circuits in left atrial macroreentrant tachycardia circulation. 2002; 105:1934-1942,

7. Jaïs, P., Matsuo, S., Knecht, S., et al. A deductive mapping strategy for atrial tachycardia following atrial fibrillation ablation: importance of localized reentry. Journal of Cardiovascular Electrophysiology, 2009, 20 (5): 480-491

8. Derejko P, Podziemski P, Zebrowski JJ, et al. Effect of the restitution properties of cardiac tissue on the repeatability of entrainment mapping response. Circ Arrhythm Electrophysiol. 2014 Jun;7(3):497-504.

9. Anter E ,Tschabrunn C, Josephson ME et al. High-resolution mapping of scar-related atrial arrhythmias using smaller electrodes with closer interelectrode spacing. Circulation: Arrhythmia and Electrophysiology. 2015; 8:537-545

10. Jaïs $P$, Hocini M, Hsu LF, et al. Technique and results of linear ablation at the mitral isthmus. Circulation. 2004, Nov 9;110(19):2996-3002.

11. Coffey JO, d\&\#39;Avila A, Dukkipati S, et al. Catheter ablation of scar-related atypical atrial flutter. EP Europace, Volume 15, Issue 3, 1 March 2013, Pages 414-419 